\title{
Littre Hernia: A Rare Case of an Incarcerated Meckel's Diverticulum
}

\section{(ㅇ)(1) $\circledast$}

\author{
Authors \\ Brian Malling1 , Andreas Aarenstrup Karlsen², \\ Jesper Hern ${ }^{3}$ \\ Affiliations \\ 1 Rigshospitalet, Department of Diagnostic \\ Radiology, Kobenhavn, Denmark \\ 2 Nordsjaellands Hospital, Surgery, Hillerod, \\ Denmark \\ 3 Nordsjaellands Hospital, Department of \\ Radiology, Hillerod, Denmark
}

\section{Background}

A Meckel's diverticulum is a remnant of the vitelline duct, which leads to the formation of a true diverticulum containing all layers of the small intestine. The diverticulum can contain ectopic gastric, duodenal or pancreatic tissue and is the most common congenital anomaly of the gastrointestinal tract with estimates of prevalence ranging from $0.3 \%$ to $3 \%$. The condition is usually clinically silent. In children the most common complication is gastrointestinal bleeding caused by ulceration due to the acid secretion by ectopic gastric mucosa. In adults, however, the most commonly observed complication is bowel obstruction followed by inflammation and bleeding (J. Dumper et al. Can J Surg 2006;49(5):353-357). In the year 1700, the French surgeon Alexis Littre was the first to describe cases of incarcerated femoral hernias containing a small bowel diverticulum. This condition is not to be confused with a Richter's hernia where only a portion of the wall is strangulated in a hernia sac (RK. Baum, IY. Olch. Calif Med 1958;88(5):386-388). The her-

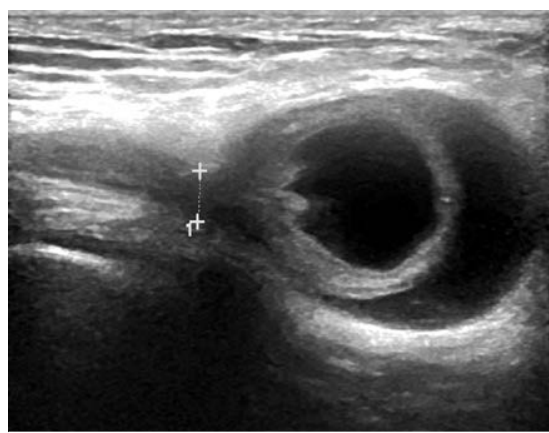

- Fig. 1 Ultrasound findings showing herniation of small intestine. niation of a Meckel's diverticulum - the socalled Littre hernia - is extremely rare and less than 50 cases have been described in the literature over the past 300 years. The anatomical sites of a Littre hernia can vary and most commonly involve the inner groin (inguinal), the outer groin (femoral) and the belly button (umbilical)(Y. Yagmur et al. J Clin Cases WJCC 2014;2(6):232-234). Incarceration of a hernia is a term used to describe entrapment of the hernia contents leading to swelling of the trapped tissue. This ultimately reduces arterial flow resulting in ischemia and necrosis of the hernia contents also termed strangulation. Such complicated groin hernias usually present with symptoms of bowel obstruction, skin changes or peritonitis and require emergency surgery, but in the extremely rare case of an incarcerated Littre hernia, the symptoms can be less severe and occur late in the course of the disease. This is because only the diverticulum and not the lumen of the intestine is involved, allowing the passage of feces. We would like to present such a rare case.

\section{Case description}

A 52-year-old woman presented with a 3-day-old hard and painful mass in the right groin. The mass had increased in size to approximately $3 \mathrm{~cm}$. The medical interview revealed no gastrointestinal symptoms. In addition no B-symptoms (fever $>39^{\circ} \mathrm{C}$, drenching, especially nightly, sweats and unintentional weight loss of $>10$ of bodyweight in 6 months or less) or symptoms of

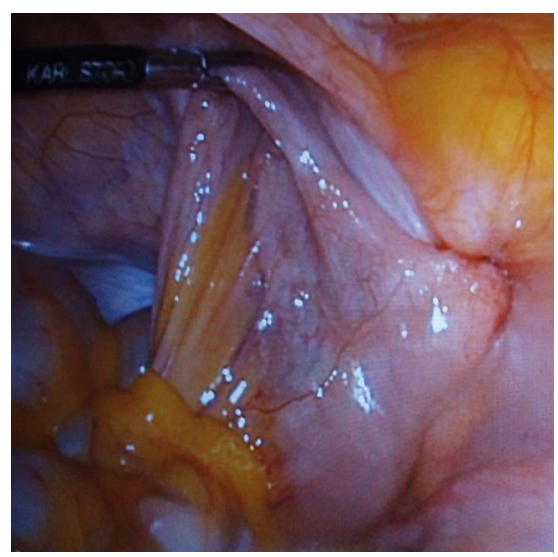

- Fig. 2 Identification of the hernia. infection were reported by the patient. During clinical examination, there was slight tenderness of the tumor, but no discoloration of the skin, abdominal pain or fever. Reduction of the tumor was not possible. Standard lab tests were inconspicuous. Suspected for malignant tumor, the patient was referred to the Department of Radiology, North Zealand Hospital for the purpose of ultrasonic characteristics of the tumor and ultrasound-guided core needle biopsy. The ultrasound examination showed a fluid-filled and blind ending structure located medially on the right thigh ( $\triangleright$ Fig. 1 ). The wall of the structure was multilayered and showed a subtle pulsatile signal during color Doppler examination. These findings suggested a femoral hernia containing small intestine. The hernia was not amenable to reduction and as such was incarcerated. Surgical repair was therefore indicated. During laparoscopic hernia repair, the hernia was identified ( $\triangleright$ Fig. 2 ) and after reduction of the hernia sac, the relevant part of the small intestine proved to be a Meckel's diverticulum ( $\mathbf{F i g}$. 3). The surgeon reported that the diverticulum had initially appeared discolored, indicating some degree of strangulation, but after reduction normal coloration was regained. The diverticulum was resected and the tissue sent for further examination. Histology later showed signs of inflammation and fibrosis. No ectopic mucosa, which may be seen in a Meckel's diverticulum, was present.

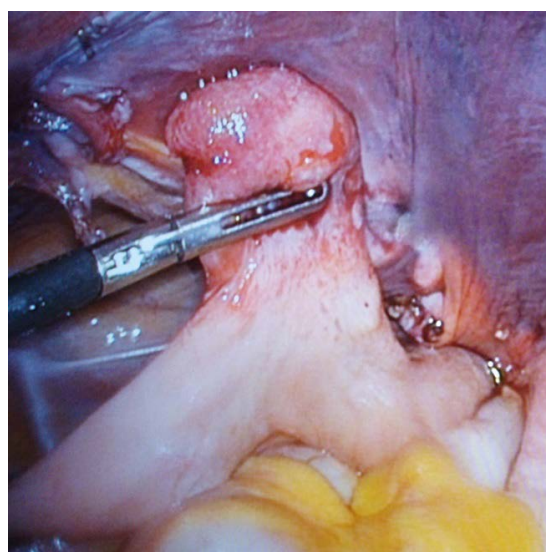

- Fig. 3 Reduction of a Meckel's diverticulum 


\section{Discussion}

In women femoral hernias constitute 34\% of all hernias and because of the narrow and rigid femoral canal the contents incarcerate or strangulate in up to $60 \%$ of cases (P. Misiak et al. Przeglad Gastroenterol 2014;9(3):172-174). In light of these circumstances, watchful waiting is not recommended especially in women who should undergo surgical hernia repair. Early diagnosis is essential to protect the viability of the intestine, but making the initial diagnosis of groin hernia can be complicated by other diseases presented as a groin tumor, including great saphenous vein varicosity (saphena varix), psoas abscess, cystic masses and enlarged lymph nodes. Distinguish- ing between these conditions was, in this case, done by demonstrating simple ultrasonic characteristics of the intestine wall. Teaching emergency physicians simple ultrasound skills could help to avoid unnecessary invasive interventions, e. g. biopsy or groin exploration, or as pointed out above, accelerate the time to surgery.

In this case, a potential fatal condition ( $R$. Tse, RW. Byard J Forensic Sci 2012;57(1):260261) with incarceration of the small intestine did not show typical symptoms of strangulation even though some impairment in intestinal circulation was evident. Ultrasound is a widely available, noninvasive and cheap examination method which in this case provided important initial information and al- lowed fast and relevant treatment of what proved to be a rare case of Littre hernia.

\section{Conflict of Interest}

The authors declare that they have no conflict of interest.

Correspondence

Dr. Brian Malling, MD

Rigshospitalet

Department of Diagnostic Radiology

Blegdamsvej 9

2100, Kobenhavn

Denmark

Tel.: + 45/354/53024

brianmalling@icloud.com 